\title{
Metilfenidato como auxiliar en pacientes con ansiedad social que reciben escitalopram
}

\author{
Rafael Jesús Salin-Pascual1,2, Nayely Fuentes-Romero y Andrea Arroyo-Guzmán ${ }^{1}$ \\ ${ }^{1}$ Departamento de Psiquiatría, Facultad de Medicina, Universidad Nacional Autónoma de México; ${ }^{2}$ Investigador nacional del Sistema Nacional de \\ Investigadores. Ciudad de México, México.
}

\begin{abstract}
Resumen
La ansiedad social (AS) es uno de los trastornos por ansiedad de más alta prevalencia a nivel mundial. Los tratamientos de elección se centran en los inhibidores de recaptura de serotonina (ISRS), entre los cuales se encuentra el escitalopram (ES). Una estrategia no farmacológica consiste en la exposición graduada a las tareas que el paciente evita, por temor a quedar en ridículo, haciéndolo gradualmente como parte de la terapia cognitivo-conductual. Si bien el tratamiento con ISRS disminuye la ansiedad anticipatoria, no favorece que los pacientes se expongan a las tareas programadas. El metilfenidato es un medicamento utilizado comercialmente en el trastorno por atención deficiente que tiene un aumento del tono dopaminérgico. En el presente estudio se evaluaron dos grupos de pacientes con AS: a un grupo se le administró ES (10 mg/día) (grupo ES) y al otro una combinación de ES (10 $\mathrm{mg} / \mathrm{día}$ ) y metilfenidato (20 $\mathrm{mg}$ repartido en dos tomas, al despertar y antes de comer) (grupo MFES) de manera abierta. Se evaluó el cambio en la escala de AS de Lebowitz y en la escala de Hamilton para ansiedad al inicio del tratamiento y ocho semanas después. Se siguió a los pacientes durante cuatro consultas, con dos semanas de separación, y se les pidió que completaran una serie de tareas de exposición. El estudio fue abierto y se aplicó estadística no paramétrica para evaluar los resultados. El principal objetivo de este estudio abierto fue evaluar si el efecto reforzador del metilfenidato podría facilitar el desempeño de la exposición graduada a los estímulos generadores de AS. Se estudiaron 15 pacientes. No hubo diferencia en la calificación basal de la escala de Liebowitz ni en las escalas Hamilton de ansiedad (HAM-A) basales. El grupo MFES mostró diferencias significativas en la escala de Leibowitz para la AS (pretratamiento: $33.29 \pm 2.87$ vs. postratamiento: $13.71 \pm 3.5$; prueba del signo de Wilcoxon $p<0.031$ ), mientras que en el grupo ES las diferencias no fueron significativas (pretratamiento: $32.15 \pm 5.2$ vs. postratamiento: $24.1 \pm 8.4 ; p=0.68$ ). Lo mismo se observó en la escala de HAM-A. El grupo MFES mostró diferencias entre los estados de pretratamiento $(21.85 \pm 7.0)$ y postratamiento $(9.14 \pm 1.95)(U$ de Mann-Whitney $p<0.031)$, pero no el grupo ES (pretratamiento: $26.25 \pm$ 7.04; postratamiento: $21.25 \pm 7.4$; prueba de Signo de Wilcoxon $p=0.125$ ). También hubo diferencias a nivel de las tareas de exposición en el grupo MFES a lo largo de las ocho semanas del estudio, mientras que las diferencias del grupo ES fueron menos significativas. Así pues, se puede concluir que, si bien la monoterapia con ISRS es eficaz, como ha sido demostrado en estudios previos en AS, la adición del metilfenidato puede acortar el tiempo de exposición a las tareas que los pacientes evitan, con lo cual se puede lograr un alivio en menos tiempo. Se requieren estudios controlados con paradigmas parecidos antes de realizar mayores conclusiones al respecto.
\end{abstract}

Palabras clave: Ansiedad social. Fobia social. ISRS. Metilfenidato. Terapia cognitivo-conductual.

\section{Correspondencia:}

Rafael Jesús Salin-Pascual

Jojutla 37, Casa 7

Col. La Joya, Del. Tlalpan
Fecha de recepción: 20-01-2018

Fecha de aceptación: 04-10-2018

E-mail: rafasalinpas@gmail.com DOI: 10.24875/RMN.M18000013

1665-5044/@ 2018. Academia Mexicana de Neurolo

(http://creativecommons.org/licenses/by-nc-nd/4.0/)
Disponible en internet: 03-01-2019 Rev Mex Neuroci. 2018;19(6):12-19 www.revmexneurociencia.com A.C. Publicado por Permanyer México. Este es un artículo Open Access bajo la licencia CC BY-NC-ND 


\title{
Methylphenidate as an aid in patients with social anxiety receiving escitalopram
}

\begin{abstract}
Social anxiety (SA), is one of the top anxiety disorders, with highest prevalence worldwide. Main treatments is focus on Serotonin Selective Recapture Inhibitors (SSRIS), like escitalopram. The other nonpharmacological strategy is graduated exposure to a series of particular tasks that the patient avoids, for fear of being ridiculized. That estrategy is part of cognitive behavioral therapy. Treatment with SSRIs, although decreases anticipatory anxiety, does not favor that patients could be exposed to some scheduled tasks. Methylphenidate is a drug that is used commercially in attention deficit disorder, and has increased dopaminergic tone, specially in some reward regions of the brain. The main objetive of this open study was to figure out if the reward stimulation of methylphenidate could improve the exposure to SA patientes to social activities that otherwise they avoided at all. In this study two groups SA of patients were evaluated. Both of them were administered escitalopram $10 \mathrm{mg} / \mathrm{daily}$, and in one group the combination of escitalopram $10 \mathrm{mg} / \mathrm{day}$ and methylphenidate (20mg/day - $10 \mathrm{mg}$ Early in the morning and $10 \mathrm{mg}$ before lunch). Change were evaluated on both Lebowitz social anxiety scale and Hamilton anxiety scale, at baseline and 8 weeks later. Each patient was followed along four consultations, two weeks apart and were asked to complete a series of tasks exposure related of the things that they avoided. The study was open, and nonparametric statistics was applied to evaluate the results. Fiveteens patients were studied, divided into two groups, those with methylphenidate (MPES) and those without (ES). Both groups received escitalopram $10 \mathrm{mg} / \mathrm{d}$. There were no difference in basal rating scale or scales Liebowitz and HAM-A, at basline. The methylphenidate group and escitalopram showed significant differences in Leibowitz at the end of the study. Pretreatment MPES: $33.29+$ 2.87; postreatment MPES $13.71+3.5$ (Wilcoxon sign test $p<0.031$ ). While in the group with monotherapy, ES Pretreatment and Postreatment were not significant $(32.15+5.2$ vs. $24.1+8.4, p=0.68)$. The same was observed in the HAM-A, MPES showed differences between baseline states $21.85+7.0$ and posttreatment $9.14+1.95$ (U-Mann-Whitney $p<0.031$ ) but not in the group with escitalopram monotherapy, pre: $26.25+7.04$ and $21.25+7.4$ post (Wilcoxon test $p=$ sign 0.125$)$. There were also differences in the level of tasks exposure in the MPES group over the eight-week study, while differences in the monotherapy group were significant but smaller. It can be concluded that although monotherapy SSRI is effective, as has been shown in previous studies in SA; the addition of methylphenidate may shorten the exposure time to the tasks that regularly are avoided by the patients, so that relief can be achieved in less time. They require similar paradigms controlled before major conclusions about studies.
\end{abstract}

Key words: Social Anxiety. Social Phobia. SSRIs. Methylphenidate. Cognitive behavioral therapy.

Estudio piloto del proyecto «Estudio doble ciego y controlado de la respuesta en ansiedad social ante el manejo farmacológico con escitalopram y metilfenidato versus escitalopram y placebo", número 066/2016;

Facultad de Medicina, UNAM.

\section{Introducción}

La AS se reconoció como tal en la década de 1960, se incorporó al manual de clasificación y estadística de los trastornos mentales, de la American Psychiatric Association, en su tercera edición (DSM-III), en la década de 1980 se diferenció la fobia social del resto de fobias simples y en la cuarta edición del mismo manual de la American Psychiatric Association se modificó el nombre por el de ansiedad social, que incluye temor y malestar físico relacionados con la mayoría de las situaciones sociales. Esta terminología persiste en el actual manual DSM-5.

\section{Síntomas y signos de la ansiedad social}

El síntoma central es un temor excesivo a situaciones sociales o a ejecutar algún tipo de actividad frente a personas fuera del círculo cercano familiar o de ámbitos sociales en los que el paciente acostumbra a convivir, aunque en las formas extremas, denominadas "ansiedad social generalizada", incluso se evita este tipo de interacción con la propia familia. La persona tiende a avergonzarse por percibir de los demás, de manera sesgada, una evaluación negativa ${ }^{2}$.

Hablar en público es una de las situaciones que genera ansiedad en cualquier persona, aun sin fobia social, y ésta es la situación que se refiere como la más común de todos los síntomas en pacientes con AS. Otras situaciones temidas por la mayoría de estos pacientes incluyen interacciones informales con personas, como ir a fiestas, reuniones o salir en una cita amorosa, las conductas afectivas como el platicar con personas de autoridad o expresar un desacuerdo, y situaciones que involucran cierto grado de escrutinio, tales como trabajar y ser observado. Algunos síntomas de la fobia social menos frecuentes son el temor a las pruebas 0 exámenes de tipo oral (con sinodales o exposición de temas en comités de tipo tutorial), comer enfrente de otras personas, utilizar teléfonos públicos, trabajar, leer o escribir cuando se es observado (cabe la posibilidad de que no se le observe del todo, pero ésta es un área 
de distorsión cognitiva en la que las personas perciben ser el centro de los eventos sociales), utilizar los baños públicos o cualquier situación que implique la interacción social con desconocidos. En casos extremos, por ejemplo, en la llamada «ansiedad social generalizada», hay un temor y evitación para cualquier situación social que involucre una interacción con los demás².

Hay que subrayar que, aun cuando el paciente evite vincularse socialmente, existe un conflicto interior, pues necesita y desea este tipo de vinculación social, pero el grado de molestias de tipo neurovegetativo con la sola evocación o imaginarse que está en una situación social le activa las reacciones de "ataque o huida», y ha aprendido a usar la segunda opción como la llamada «zona de confort». Esto último genera una diferencia significativa con otras formas severas de evitación social, como la personalidad esquizoide, las personalidades esquizotípica y la personalidad pasivo-agresiva ${ }^{3}$.

Los síntomas de la AS se pueden categorizar como somáticos, cognitivos y conductuales. El enrojecimiento y la sudoración están dentro de los síntomas más comunes de tipo somático, junto con las palpitaciones, temblor de manos, malestar abdominal y tensión muscular. Los síntomas de ansiedad pueden ser tan severos que pueden convertirse en un ataque de pánico, de modo que esta entidad clínica es una de las comorbilidades más frecuentes, pero se diferencia del trastorno por ataque de pánico como tal porque este último no es predecible, mientras que los activados por la AS sí y ocurren en un contexto de exposición social generador de la ansiedad. Los síntomas cognitivos y emocionales incluyen pensamientos desagradables acerca de la situación en la que se encuentran, habitualmente con un tipo de distorsión cognitiva que tiende a exagerar el malestar. Hay una sensación de que la gente los critica o habla mal de ellos. Esto los lleva a síntomas emocionales que pueden bloquear el flujo del pensamiento y la concentración. Las personas con fobia social muy a menudo temen que otras personas descubran lo que les sucede. Por ejemplo, que detecten su nerviosismo, sudoración, enrojecimiento, malestar y temblor. También temen que otros puedan cuestionar o criticar su situación y juzgarlos como estúpidos o ignorantes. Paradójicamente, esto les impide a la larga el practicar y desensibilizarse dentro de las situaciones que temen, y ganar al mismo tiempo experiencia y confianza social. Algunas de las situaciones que con más frecuencia evitan los pacientes con AS se muestran en la tabla $1^{4}$.

Es común que la AS se asocie con una ansiedad anticipatoria. Por ejemplo, en la medida en que se aproxima la fecha de un evento determinado, en el que se tendrá que exponer o hacer una ejecución frente a un grupo de gente, aumentará el tipo de ansiedad anticipatoria, lo cual puede llevar a estados de congelamiento o de evitación del evento. Al cabo de lo cual, cualquiera que haya sido la decisión tomada, si el resultado es negativo, reforzará la percepción de invalidez social y la autoestima disminuirá. A la larga se puede establecer un cuadro de depresión o un cuadro clínico mixto con ansiedad y depresión, los cuales exacerban el malestar ante los eventos sociales ${ }^{4}$.

\section{Subtipos de trastorno por ansiedad social}

Para algunos investigadores, en la AS existe un espectro similar al de otros trastornos psiquiátricos, denominado «espectro del trastorno por ansiedad social», en el que hay una variación en la percepción de esta condición vinculada con aspectos culturales. Es importante tomar en cuenta que, para algunos críticos del sistema de clasificación psiquiátrica, la AS es simplemente un extremo de las capacidades de vinculación que presentan los seres humanos. Éstas van desde las personas extrovertidas e histriónicas hasta el tipo de AS generalizada. Sin embargo, las demandas sociales en los complejos urbanos, universidades, consorcios y comercios exigen la interacción social afectiva como un valor personal ${ }^{5-7}$.

Dentro del espectro de la AS encontramos en primer lugar un rasgo de carácter que se denomina «timidez». Aunque la mayoría de las personas experimenten periodos de timidez (cerca del $90 \%$ de los individuos en autorreportes expresan haber sentido timidez alguna vez en su vida), ésta no es sinónimo ni equivalente de fobia social, ya que sólo una minoría de ellas desarrollarán AS. En las escalas que miden el rasgo de personalidad de timidez, cuando están en el rango de mayor severidad, el $49 \%$ fueron también diagnosticadas con AS. Es posible encontrar una relación entre timidez y AS, pero no son sinónimos, pues una persona puede ser extremadamente tímida sin sufrir AS. La principal diferencia radica en que en la segunda alteración se presenta ansiedad anticipatoria ante un evento en el que hay una probabilidad de exponerse en un contexto social.

El surgimiento del temor en los niños hacia los extraños se observa entre los 7 y 10 meses de edad; es una respuesta considerada normal y disminuye con el tiempo ${ }^{8}$. En términos ontogenéticos, se ha propuesto que es normal que los niños de 7 a 10 meses tengan temor a los extraños, pues esto genera reacciones de alarma hacia sus padres o cuidadores. Sin embargo, si persiste y se asocia con temor a situaciones no familiares en la infancia, se convierte en un predictor 
Tabla 1. Ejemplos de situaciones que el paciente evita

\begin{tabular}{|c|c|c|c|c|}
\hline Situaciones que evita & Calificar del 0 al 10 & Muy importante & Importante & No importante \\
\hline \multicolumn{5}{|l|}{ Contestar el teléfono o hablar por él } \\
\hline \multicolumn{5}{|l|}{ Contestar a preguntas en clase } \\
\hline \multicolumn{5}{|l|}{ Pedir a alguien que salga con él } \\
\hline \multicolumn{5}{|l|}{ Preguntar algo al maestro o pedir ayuda } \\
\hline \multicolumn{5}{|l|}{ Ir a fiestas, bailar 0 actividades sociales } \\
\hline \multicolumn{5}{|l|}{ Enrojecimiento, temblor o sudoración frente a otras personas } \\
\hline \multicolumn{5}{|l|}{ Comer con desconocidos } \\
\hline \multicolumn{5}{|l|}{ Entrar a una habitación donde hay otras personas } \\
\hline \multicolumn{5}{|l|}{ Dar un reporte o leer delante de otras personas } \\
\hline \multicolumn{5}{|l|}{ Salir en una cita } \\
\hline \multicolumn{5}{|l|}{ Invitar a un amigo a salir } \\
\hline \multicolumn{5}{|l|}{ Permitir que le hagan una fotografía } \\
\hline \multicolumn{5}{|l|}{ Participar en un grupo o en clases } \\
\hline \multicolumn{5}{|l|}{ Actuar en público } \\
\hline \multicolumn{5}{|l|}{ Hablar a una figura de autoridad } \\
\hline \multicolumn{5}{|l|}{ Iniciar o unirse a una conversación } \\
\hline \multicolumn{5}{|l|}{ Hacer un examen escrito u oral } \\
\hline \multicolumn{5}{|l|}{ Hablar a una figura de autoridad } \\
\hline \multicolumn{5}{|l|}{ Ser evaluado } \\
\hline \multicolumn{5}{|l|}{ Utilizar baños públicos } \\
\hline \multicolumn{5}{|l|}{ Caminar por pasillos con gente desconocida } \\
\hline \multicolumn{5}{|l|}{ Trabajar con grupos de adolescentes } \\
\hline \multicolumn{5}{|l|}{ Escribir en el pizarrón delante de otras personas } \\
\hline Otras actividades & & & & \\
\hline
\end{tabular}

temprano de fobia social. El mutismo selectivo en la infancia es equivalente a la AS en niños y adultos. En esta condición, los niños sólo hablan con uno de sus padres o hermanos, pero no se comunican con los demás miembros de la familia. El $100 \%$ de niños con mutismo selectivo desarrollan AS y el $70 \%$ de los familiares de primer grado tienen algunas formas de AS dentro del espectro antes mencionado ${ }^{9,10}$.

Se ha propuesto también que parte de la psicopatología de la AS se deba a una excesiva preocupación por la imagen corporal, sin que llegue a los extremos de la dismorfofobia (los pacientes perciben zonas de su rostro o cuerpo deformes), pero sin claras tendencias a la excelencia social o perfeccionismo social, en los que no se permiten tener pequeños errores 0 defectos, lo cual les lleva a que se estén monitorizando continuamente.

El ES ha demostrado ser útil en la $\mathrm{AS}^{11-13}$ con un buen margen de seguridad y eficacia, pero como parte de la terapia cognitivo-conductual, en la que se pide a los pacientes con AS que se expongan a situaciones que han evitado como parte de su enfermedad y que las lleven a cabo, siendo un obstáculo a vencer.

Existe un reporte en la literatura médica reciente del uso del metilfenidato como monoterapia en la AS en dos pacientes adultos que presentaban además trastorno por atención deficiente ${ }^{14}$. El antidepresivo ISRS mejora el estado de ansiedad anticipatoria que hace que los pacientes eviten exponerse; sin embargo, no implica que realicen sus tareas como parte de la 
terapia cognitivo-conductual. Esto es, el componente motivacional motor se puede amplificar con este estimulante del sistema nervioso central.

El perfil farmacológico del metilfenidato es el de un estimulante con una potencia intermedia entre la cafeína y la cocaína ${ }^{15}$. Este efecto se logra por un bloqueo de los transportadores de dopamina y norepinefrina (proteínas de recaptura que remueven estos neurotransmisores de la hendidura sináptica ${ }^{16,17}$. Al aumentar el tiempo de exposición de ambas catecolaminas, se refuerza el efecto placentero de ciertas acciones.

Los pacientes con AS sí tienen deseo de participar en fiestas, discusiones, aproximarse a personas que les resultan atractivas..., pero una serie de disfunciones cognitivas los llevan a anticiparse a la posibilidad de fracasar y la probabilidad de resultar ridículos o ser rechazados se lo impide. Es por esto que se propone que un agente como el metilfenidato pueda ser de utilidad en el tratamiento de la AS.

\section{Objetivos}

Evaluar si el aumento de metilfenidato a un tratamiento convencional con un ISRS produce mejores resultados clínicos y en la terapia cognitivo-conductual en pacientes con AS.

\section{Método}

Se estudiaron 15 pacientes con diagnóstico de AS, según los criterios del DSM-IV-TR ${ }^{18}$, en un protocolo abierto de asignación de aumento de tratamiento a un ISRS, el ES más el metilfenidato: grupo MFES y grupo ES. La asignación a cada una de las ramas de tratamiento se hizo mediante una tabla de números aleatoria.

Todos los pacientes fueron evaluados con la entrevista estructurada para el diagnóstico psiquiátrico del eje 1 del DSM-IV-TR ${ }^{19}$. La escala de Liebowitz para la AS contiene 24 reactivos y 2 columnas para evaluar si el reactivo preguntado produce ansiedad y qué tanto evita de ser posible enfrentar lo que se le pregunta, pues una persona puede tener mucha ansiedad para presentarse a exámenes pero, si está en la universidad, no podrá evitarlo. También se empleó la escala de Hamilton para la ansiedad de 15 reactivos. Los pacientes fueron evaluados cada 2 semanas durante un periodo de 8 semanas. Los pacientes recibieron ES $10 \mathrm{mg} / \mathrm{día}$ (Selective, Psicofarma) y ajustaron su toma al desayuno o cena según si manifestaban somnolencia o no. Esta condición es idiopática y sólo se pidió que fuera notificada. Los enfermos con metilfenidato recibieron siempre $10 \mathrm{mg}$ por la mañana, al despertar, y otra tableta de $10 \mathrm{mg}$ al mediodía (Ritalin, Novartis).

Con cada uno de los pacientes se elaboró una hoja personalizada de tareas a las que se tendrían que exponer gradualmente. En la tabla 1 se ve un ejemplo de esta herramienta. Se empezó con las tereas a las que ya se podían exponer, aunque con cierta ansiedad, que en la hoja de tareas de exposición correspondía a calificaciones de 5 a 6 . A todos los pacientes se les entrenó en maniobras de relajación, utilizando las técnicas de respiración y de contar mentalmente del uno al cuatro, en la inhalación, sostener el aire y expiración, así como en ejercicios de relación isométrica. Esta tarea se hizo dos veces al día durante las ocho semanas del estudio. A los pacientes se les pidió que hicieran estos ejercicios de relajación antes de enfrentarse a su exposición graduada.

En cada visita se calificó cada una de las exposiciones logradas con el número de la escala analógica visual que obtuvieron en las tareas a exponerse en las situaciones generadoras de AS. La exposición repetida a estas situaciones se calificó restando puntos a sus calificaciones basales de cada reactivo de la escala Liebowitz. A las ocho semanas se volvieron a aplicar las escalas de Liebowitz para la AS y la de Hamilton para la ansiedad, y se rellenó una hoja de exposición graduada con el avance máximo logrado. La estadística que se empleó fue la no paramétrica.

\section{Resultados}

Se estudiaron 15 pacientes: 5 mujeres y 10 hombres; sus datos demográficos se muestran en las tablas 2 y 3 . Los pacientes del grupo MFES tenían todos el diagnóstico de AS generalizada, mientras que en el grupo ES cuatro de ocho presentaban la forma no generaliza. Sin embargo, en cuanto a la calificación basal de la escala de Liebowitz no hubo diferencias entre el grupo MFES $(33.29 \pm 2.87)$ y el grupo ES $(32.12 \pm 5.24)$. Tampoco hubo diferencias en las escalas de HAM-A basales: grupo MFES $=21.85 \pm 7.03$ y grupo ES $=26.25 \pm 7.04$.

La comparación entre los valores basales de la escala de Leibowitz y después del tratamiento en el grupo MFES mostró diferencias significativas (pretratamiento de $33.29 \pm 2.87$ vs. postratamiento de $13.71 \pm 3.5$; prueba del signo de Wilcoxon $p<0.031$ ), mientras que en el grupo ES las diferencias pretratamiento y postratamiento no fueron significativas después de ocho semanas $(32.15 \pm 5.2$ vs. $24.1 \pm 8.4$, respectivamente; prueba del signo de Wilcoxon $p=0.68$ ). 
Tabla 2. Grupo de MFES

\begin{tabular}{|l|l|l|c|}
\hline Paciente & Diagnóstico & Género & Edad \\
\hline AA & FS generalizada & Masculino & 40 \\
\hline AJ & FS generalizada & Masculino & 52 \\
\hline EE & FS generalizada & Femenino & 42 \\
\hline FP & FS generalizada & Masculino & 40 \\
\hline AP & FS generalizada & Masculino & 22 \\
\hline GE & FS generalizada & Masculino & 30 \\
\hline
\end{tabular}

FS: Fobia social.

\begin{tabular}{|l|l|l|c|}
\hline Paciente & Diagnóstico & Género & Edad \\
\hline BC & FS generalizada & Masculino & 25 \\
\hline GC & FS generalizada & Femenino & 45 \\
\hline NC & FS no generalizada & Femenino & 30 \\
\hline RH & FS no generalizada & Masculino & 25 \\
\hline MR & FS generalizada & Masculino & 38 \\
\hline MP & FS generalizada & Femenino & 20 \\
\hline GL & FS no generalizada & Femenino & 40 \\
\hline AR & FS no generalizada & Femenino & 32 \\
\hline
\end{tabular}

La escala de Hamilton para la ansiedad en el grupo MFES mostró diferencias entre el pretratamiento (21.85 $\pm 7.0)$ y el postratamiento $(9.14 \pm 1.95)$ (prueba de la $U$ de Mann-Withney $p<0.031$ ), pero no en el grupo ES: pretratamiento de $26.25 \pm 7.04$ y postratamiento de $21.25 \pm 7.4$ (prueba de signo de Wilcoxon $p=0.125$ ).

En la figura 1 se puede apreciar la reducción de la puntuación vinculada a las tareas de exposición. Al inicio del estudio se dio una calificación de 10 a cada uno de los cinco eventos generadores de ansiedad particulares a cada paciente (hablar en público, pasar al pizarrón, conocer a gente nueva, comer solo con personas desconocidas, etc.), por eso se partió de 50 puntos como calificación total, sobre los cuales se podría observar una disminución a lo largo del estudio. Existió una diferencia significativa en los pacientes del grupo MFES desde la segunda visita del estudio (un mes), en comparación con el grupo ES; mejoría que prosiguió hasta la octava semana.

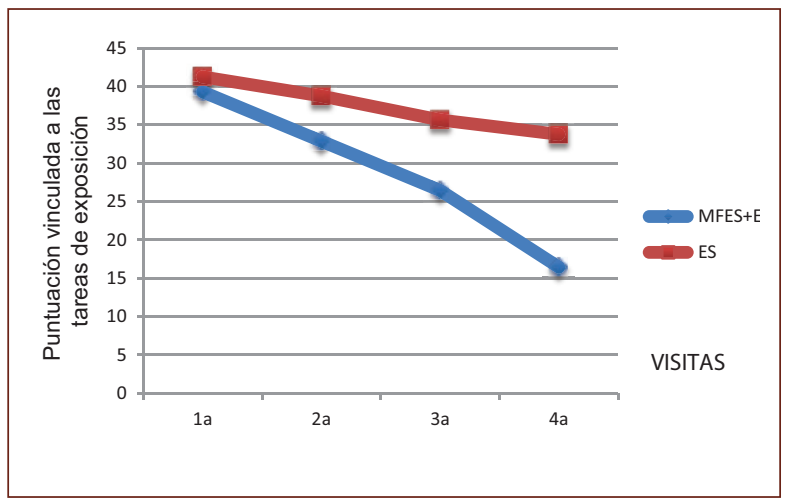

Figura 1. Cambios en la puntación de las tareas ansiogénicas.

Aun cuando ambos grupos mostraron una reducción en sus tareas a exponerse, en el caso del grupo MFES fue de significancia estadística, esto es, más del $50 \%$ de sus valores basales (prueba de Friedman $\chi^{2}$ de 21.0; $\mathrm{Gl}=3 ; p<0.0001$ ), mientras que en el grupo ES la respuesta fue moderadamente significativa a lo largo de las cuatro primeras semanas de seguimiento (prueba de Friedman $p<0.01$ ). Si se toma el criterio de respuesta al tratamiento como la reducción del $50 \%$ de los valores de ingreso al estudio, encontramos que en el grupo MFES la respuesta fue del $100 \%$ para la octava semana (siete de siete pacientes), mientras que en el caso del grupo ES ésta fue del $62 \%$, sin que los respondedores correspondan en particular a alguno de los dos subtipos de AS estudiados (generalizada vs. no generalizada). Los efectos secundarios reportados por los pacientes fueron mínimos para el ISRS -náuseas por tres días máximo- y para el metilfenidato -nerviosismo dos días máximo-. Ningún paciente pidió ser retirado del estudio.

\section{Discusión}

La respuesta y la mejoría de los pacientes con AS con la combinación MFES fueron significativamente superiores en los parámetros estudiados. En un estudio previo con metilfenidato como monoterapia se encontró una buena respuesta en dos personas con AS y atención deficiente en la etapa adulta ${ }^{14}$. Ninguno de los pacientes del presente estudio había sido diagnosticado con atención deficiente con o sin hiperactividad a lo largo de su vida.

En un metaanálisis reciente en el que se evaluaron estudios con ES en AS que fueran doble ciego, 
aleatorizados contra placebo y en los que se empleara la escala de Leibowitz, del total de 1,061 pacientes, en 12 semanas este ISRS fue superior al placebo en todas las dosis utilizadas ${ }^{20}$. Esta situación se presentó en nuestro estudio, en el que sí hubo una mejoría de la AS en la monoterapia, pero no significativa para el periodo evaluado de dos meses, aunque sí se observó una mayor exposición a las tareas que los pacientes evitaban moderadamente significativa. Es posible que el aumento de la dosis del ISRS hubiera sido una alternativa, pues el comportamiento de estos medicamentos para el manejo de alteraciones por ansiedad sí tiene una relación lineal del tipo dosis-respuesta, diferente que en la depresión.

En niños con AS y atención deficiente e hiperactividad, de edades entre 8 y 18 años, que recibieron monoterapia con metilfenidato también se encontró una respuesta de disminución de la severidad de la AS, a la par que las manifestaciones de atención deficiente ${ }^{21}$, en donde el efecto ansiolítico parece ser paradójico, con un agente de activación dopaminérgica, pero también se ha reportado con atomoxetina ${ }^{22}$.

Los efectos del MP fueron euforia, aumento de la concentración y mayor actividad psicomotriz, lo cual pudo contribuir a que los pacientes pudieran decidirse a ejecutar sus tareas de exposición ante eventos ansiogénicos para ellos. Este efecto fue notable desde la segunda visita en el grupo MFES. En cambio, lo que reportaban los pacientes del grupo ES fue que se redujo la ansiedad, pero no se sintieron motivados para exponerse a sus tareas en particular.

El metilfenidato, como la mayoría de los estimulantes, produce un aumento en la autoconfianza, lo cual, aunado a la baja ansiedad por el ISRS, puede tener un papel reforzador en sus exposiciones en el paciente con AS. Este efecto reforzador ya se había reportado previamente ${ }^{16}$.

Existe una zona de discusión con respecto a la AS que se refiere a qué parámetros utilizar para calificar a un paciente como respondedor o con una remisión. Si bien en la depresión mayor sí hay consenso -escala de Hamilton para depresión con menos de 7 puntos-, no existe algo similar en la AS, por lo que en un metaanálisis sobre el tema se concluye que en la AS la respuesta es un parámetro suficiente, aunque quizás no el más adecuado ${ }^{23}$.

La principal limitación del presente trabajo fue que se trató de un estudio abierto y con un número reducido de pacientes. La otra limitante fue la posibilidad de un efecto adictivo; sin embargo, en las dosis administradas y por el tiempo limitado, éste no ha sido un problema en los pacientes con AS. Se está trabajando en un estudio controlado con medición de una serie de variables y un reto con oxitocina. Todo ello puede ahondar nuestro conocimiento en el tratamiento de este tipo de alteraciones que limitan las capacidades de las personas que lo padecen, pues en un mundo globalizado y tan competitivo como el nuestro tener AS es un serio limitante para el disfrute de una buena calidad de vida.

\section{Conclusiones}

El aumento del metilfenidato al tratamiento farmacológico de elección en la AS, como los ISRS y en particular el ES, mostró ser eficaz en términos de exposición a las tareas de la terapia cognitivo-conductual y de la atenuación de la ansiedad asociada a esta condición.

\section{Bibliografía}

1. McKay D. Current perspectives on the anxiety disorders: implications for DSM-V and beyond. Nueva York: Springer; 2009.

2. Robinson TM. Social anxiety: symptoms, causes, and techniques. Nueva York: Nova Science Publishers; 2010

3. Brunello N, den Boer JA, Judd LL, Kasper S, Kelsey JE, Lader M, et al. Social phobia: diagnosis and epidemiology, neurobiology and pharmacology, comorbidity and treatment. J Affect Disord. 2000;60(1):61-74.

4. Carre A, Gierski F, Lemogne C, Tran E, Raucher-Chene D, Bera-Potelle C, et al. Linear association between social anxiety symptoms and neural activations to angry faces: from subclinical to clinical levels. Soc Cogn Affect Neurosci. 2014;9(6):880-6.

5. Liebowitz MR, Ninan PT, Schneier FR, Blanco C. Integrating neurobiology and psychopathology into evidence-based treatment of social anxiety disorder. CNS Spectr. 2005;10(10):suppl13 1-11; discussion 12-13; quiz 14-5

6. Thornton-Wells TA, Avery SN, Blackford JU. Using novel control groups to dissect the amygdala's role in Williams syndrome. Dev Cogn Neurosci. 2011;1(3):295-304.

7. Morris CA. The behavioral phenotype of Williams syndrome: A recognizable pattern of neurodevelopment. Am J Med Genet C Semin Med Genet. 2010;154C(4):427-31.

8. Ginsburg GS, Grover RL. Assessing and treating social phobia in children and adolescents. Pediatr Ann. 2005;34(2):119-27.

9. Cholemkery H, Mojica L, Rohrmann S, Gensthaler A, Freitag CM. Can autism spectrum disorders and social anxiety disorders be differentiated by the social responsiveness scale in children and adolescents? J Autism Dev Disord. 2014;44(5):1168-82.

10. Beidel DC, Turner SM, American Psychological Association. Shy children, phobic adults: nature and treatment of social anxiety disorder. 2. ${ }^{-}$ed. Washington, DC: American Psychological Association; 2007.

11. Lele M, Joglekar A. Escitalopram for social anxiety disorder. Br J Psychiatry. 2005;187:290-1; author reply 291-2.

12. Kasper S, Stein DJ, Loft H, Nil R. Escitalopram in the treatment of social anxiety disorder: randomised, placebo-controlled, flexible-dosage study. Br J Psychiatry. 2005;186:222-6.

13. Asakura S, Hayano T, Hagino A, Koyama T. A randomized, double-blind, placebo-controlled study of escitalopram in patients with social anxiety disorder in Japan. Curr Med Res Opin. 2016;32(4):749-57.

14. Koyuncu A, Celebi F, Ertekin E, Kahn DA. Extended-release Methylphenidate Treatment and Outcomes in Comorbid Social Anxiety Disorder and Attention-deficit/Hyperactivity Disorder: 2 Case Reports. J Psychiatr Pract. 2015;21(3):225-31.

15. Crunelle CL, van den Brink W, Dom G, Booij J. Dopamine transporter occupancy by methylphenidate and impulsivity in adult ADHD. Br J Psychiatry. 2014;204(6):486-7. 
16. Volkow ND, Fowler JS, Wang GJ, Ding YS, Gatley SJ. Role of dopamine in the therapeutic and reinforcing effects of methylphenidate in humans: results from imaging studies. Eur Neuropsychopharmacol. 2002 12(6):557-66.

17. Volkow ND, Wang GJ, Tomasi D, Kollins SH, Wigal TL, Newcorn JH, et al. Methylphenidate-elicited dopamine increases in ventral striatum are associated with long-term symptom improvement in adults with attention deficit hyperactivity disorder. J Neurosci. 2012;32(3) 841-9.

18. American Psychiatric Association. Task Force on DSM-IV. Diagnostic and statistical manual of mental disorders: DSM-IV-TR. 4. ${ }^{\text {a }}$ ed. Washington, DC: American Psychiatric Association; 2000.

19. Othmer E, Othmer SC. The clinical interview using DSM-IV-TR. 1. ${ }^{\text {a }}$ ed. Washington, DC: American Psychiatric Pub.; 2002.
20. Baldwin DS, Asakura S, Koyama T, Hayano T, Hagino A, Reines E, et al. Efficacy of escitalopram in the treatment of social anxiety disorder: a meta-analysis versus placebo. Eur Neuropsychopharmacol. 2016;26(6):1062-9.

21. Golubchik P, Sever J, Weizman A. Methylphenidate treatment in children with attention deficit hyperactivity disorder and comorbid social phobia. Int Clin Psychopharmacol. 2014;29(4):212-5.

22. Snircova E, Marcincakova-Husarova V, Hrtanek I, Kulhan T, Ondrejka I, Nosalova G. Anxiety reduction on atomoxetine and methylphenidate medication in children with ADHD. Pediatr Int. 2016;58(6):476-81.

23. Bandelow B, Baldwin DS, Dolberg OT, Andersen HF, Stein DJ. What is the threshold for symptomatic response and remission for major depressive disorder, panic disorder, social anxiety disorder, and generalized anxiety disorder? J Clin Psychiatry. 2006;67(9):1428-34. 